\title{
Discrimination of surface tracking patterns of gamma irradiated polymers using fractals
}

\author{
V RAJINI* and K UDAYA KUMAR \\ Department of Electrical Engineering, S.S.N. College of Engineering, Kalavakkam 603 110, India \\ ${ }^{\dagger}$ Department of High Voltage Engineering, College of Engineering Guindy, Chennai 600 005, India
}

MS received 27 January 2006; revised 7 April 2006

\begin{abstract}
The purpose of this paper is to evaluate the radiation resistance of gamma irradiated ethylene propylene diene monomer (EPDM) and to identify the pattern discriminating abilities of the surface tracking patterns. Simple objects can be described by the ideal shape primitives such as cubes, cones and cylinders. But most of the natural objects are so complex that cannot be described in terms of simple primitives. Fractals have been very successfully used to address the problem of modeling and to provide a description of naturally occurring phenomena and shapes, wherein conventional and existing mathematical models were found to be inadequate. The geometrical patterns of dielectric breakdown like electrical trees, surface discharges, and lightning are known to be of fractal in nature. These fractal patterns can be analysed numerically using fractal dimensions and lacunarity. Surface tracking occurring in HV insulation systems is a very complex phenomenon and more so are the shapes of tracking patterns. It has been fairly well established that the shapes and the underlying parameters causing tracking have a $1: 1$ correspondence and therefore, methods to describe and quantify these patterns must be explored. This paper reports preliminary results of such a study wherein 2 -d tracking patterns of gamma irradiated ethylene propylene diene monomer were analysed and found to possess fairly reasonable pattern discriminating abilities. This approach appears promising and further research is essential before any long-term predictions can be made.
\end{abstract}

Keywords. Ethylene propylene diene monomer; surface tracking; fractal dimensions; box counting; IEC-587 inclined plane test.

\section{Introduction}

This paper is aimed at fractal analysis of surface tracking patterns propagating over gamma irradiated polymeric materials under a.c. voltage using inclined plane test. Fractal theory has proven to be a very useful tool in quantifying the structure of a wide range of idealized and naturally occurring objects. The application of fractals in the field of electrical insulation commenced about a decade ago. A review of the available literature indicates that modeling of the discharge and breakdown processes such as analysis of 'electrical tree' shapes has been one of the areas to which fractals have been applied. Kudo (1998) used fractal concept for electrical trees. Candela et al (2000) used fractals for partial discharge recognition. Niemeyer et al (1984) and Kebbabi and Beroual (2006) analysed the surface discharge patterns at solid/liquid interfaces using needle plane geometry. The fractal concept was also used by Wang (2004) to quantify the cracks in the materials. Since tracking patterns resemble them, it was decided to use the fractal concept to quantify the tracking patterns. It

*Author for correspondence (harirajini@ yahoo.com) must be stressed that only electrical trees and PD patterns have been shown to be fractal objects, which does not imply that tracking patterns are fractals. To our best knowledge, none of them have investigated the possibility of using fractals for the purpose of feature extraction in surface tracking patterns. In this short communication, we report our findings during such an investigation. The fractal dimensions of tracking patterns of gamma irradiated EPDM and their correlation to their dose rates and tracking time are reported. The correlation of fractal dimension to the operating voltage, flow rate and conductivity of the pollutant ought to be thoroughly understood. Ethylene propylene diene monomer has been chosen for the present study as it has superior dielectric properties, resistance to moisture, oxygen and weather. They are widely used in electrical applications such as cable insulation, cable terminations, joints, arrestor housing and insulators. The elastometer materials used for housing of composite insulators, usually EPDM and silicone rubber differ from ceramic materials in that their surface properties may be altered significantly with age. It is well known that the tracking has been the most common cause of insulation failures in the field. During outdoor service, the surface of the insulating material is subjected to moisture and 
contamination, which leads to phenomena in the form of tracking and erosion. This effect is particularly noticeable in EPDM materials, which unlike silicone rubber, do not typically remain hydrophobic but deteriorate after approximately 2 to 8 years (Gorur et al 1990; Starr 1990). The magnitude and seriousness of this problem have been well characterized and described in numerous publications. In recent years with increasing uses of electric and electronic devices in various radioactive environments, which include atomic power research, the organic insulation materials are inevitably exposed to various kinds of radiation. Hence, it becomes essential to investigate the influence of such irradiation in insulation materials. It can be seen that the literature on irradiated polymeric insulating materials, of which there are very few (Li et al 1999; Fouracre et al 1989) are of greater relevance to the application of insulators. No literature is available on gamma irradiated EPDM and perhaps, the contribution from the authors is for the first time. In this connection, to explore the possibility of using EPDM in radiation prone areas such as nuclear power plants, space applications and to identify the influence of total dosage of irradiation on tracking and their fractal dimensions, the samples of commercially available EPDM were exposed to gamma irradiation in air using a ${ }^{60} \mathrm{Co}$ gamma-source.

\section{Sample preparation}

The commercially available EPDM in the form of sheets was cut into required dimensions of $120 \times 50 \times 3 \mathrm{~mm}$. The surface of the samples was cleaned using acetone to remove any dust or grease present on the surface and dried. These samples were kept in the gamma irradiation chamber for the required dose. The ${ }^{60} \mathrm{Co}$ gamma chamber facility available at the Indira Gandhi Centre for Atomic Research (IGCAR) Laboratory, with an irradiation volume of one litre with a dose rate of $51 \mathrm{krads} / \mathrm{h}$ was used.

\section{Experimental}

There is no standard test procedure available to test the polymeric insulation materials in radiation environments. IEC-587 has given a detailed test procedure to test polymeric samples under aggravated service conditions. It was decided to use the same for the present study. Hence, the tracking test was carried out, following the inclined plane test method, as suggested in IEC-587. The schematic diagram of the experimental set up is shown in figure 1 . The sample along with the electrodes was mounted in the inclined plane mounting. The gap distance between HV and LV electrode was maintained at $50 \mathrm{~mm}$. The top electrode was connected to $\mathrm{HV}$ source and the bottom electrode was connected to ground through $100 \Omega$ resistor to measure tracking current. The potential drop across this was measured using THS-720P oscilloscope for further analy- sis of leakage current. To simulate the pollution condition over the surface of the samples, $\mathrm{NH}_{4} \mathrm{Cl}$ was allowed to flow at a constant flow rate using peristaltic pump. $\mathrm{NH}_{4} \mathrm{Cl}$ was used as the contaminant for the present study as it aggravates heat and does not leave any residue on the surface of the sample. A standard non-ionic wetting agent TRITON-X-100 was added to the contaminant to increase the wettability of the surface. The chemical equivalent of TRITON-X-100 is octyl phenol ethoxylate. AC voltage of $5 \mathrm{kV}$ was applied as the test voltage for all the test samples. The times to failure arrived at once arc inception takes place near bottom electrode and crosses two thirds of the gap or if the complete bulk volume of the material degrades at a particular location due to arcing. Otherwise, the process lasts for $6 \mathrm{~h}$ and the experiment was properly terminated. CCD camera was used to capture the progress of tracking.

\section{Image processing of tracked patterns}

The surface tracking images obtained from the test must be digitized and need to undergo an image treatment process such as reducing the image noise before any calculation can be made. Since the image treatment can influence the estimated fractal dimension value strongly, special care has to be taken with the method used. A large number of image treatment techniques can be used and it is a very difficult task to know which to apply to our specific problem. But it is important to note that the image treatment can influence the calculated value of the dimension. In this work, the colour images of the tracked patterns are first converted to grayscale images. Gaussian filter was used for removing the noise from the image. The gaussian filter smoothes an image by calculating the weighted averages in a filter box. The most important information for the interpretation of an image is the contour of the objects.

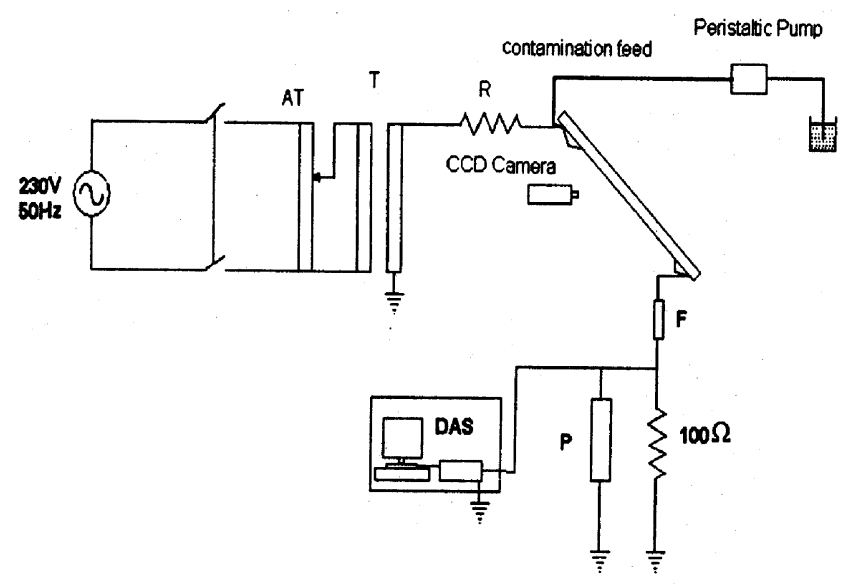

Figure 1. Inclined plane test (IEC 587) (AT, Autotransformer; T, HV transformer $5 \mathrm{kVA}, 220 \mathrm{~V} / 100 \mathrm{kV}$; R, series resistance (33 kÙ); $\mathrm{P}$, protection circuit (neon bulb); F, fuse $(100 \mathrm{~mA}))$. 
Contours are indicated by abrupt changes in brightness. A simple threshold and edge detection technique was used for treating the images and to obtain the contours.

\section{Box counting method}

The dimensions of fractal like structure (self similar) can be measured using multi resolution approach as for instance, the box counting method. Increasing the size of the box is used to explore the structure of the distribution points in a background space. Covering the figure by squares of side $L$ performs this method. The magnitude of the square is changed at each step. Thus one determines the number of squares, $N(L)$, necessary to completely cover the considered structure. The relation between the number of covering squares and the magnitude of the square side determines the fractal dimension, $D F$, according to the power law,

$$
N(L)=K L^{-D F}
$$

where $D F$ is the dimension of the fractal set. The dimension, $D F$, can be estimated from the slope of a line fit to the data $\{\log L,-\log (N(L))\}$ for several box sizes, $L$. The fractal like structure is revealed by the linearity of the plot, $\log (N(L))$ vs $1 / \log (L)$, the slope gives the fractal dimension. A sample logarithmic plot drawn for $100 \mathrm{krad}$ tracking image is shown in figure 2 . The linearity of the plot ascertains that the tracking images are fractals.

\section{Results and discussion}

The samples of EPDM were irradiated by gamma rays. The tracking test was carried out for the irradiated specimens and the tracking time was observed. The variation in the

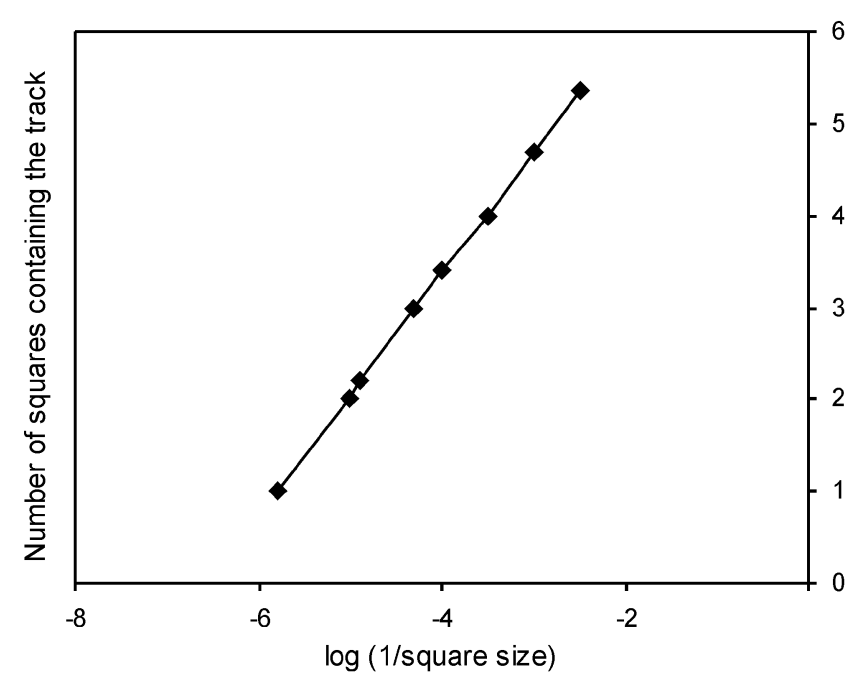

Figure 2. Sample logarithmic plot for 100-krad patterns. tracking time with variation in the total dosage is shown in figure 3. The tracking time given here are the mean of three tests conducted at the same voltage and dosage levels.

It is clear that the tracking time decreases with the increase in gamma dosage. To ascertain this again, a sample was exposed to a higher dosage of $1496 \mathrm{krad}$ and the sample failed immediately after the application of voltage in the inclined plane test. The voltage applied was $5 \mathrm{kV}$ a.c., the pollutant was $0 \cdot 1 \% \mathrm{NH}_{4} \mathrm{Cl}$ and the flow rate was kept at $0.6 \mathrm{ml} / \mathrm{min}$ for all the samples. Figure 4 shows a typical picture taken during the progress of tracking using CCD camera.

\subsection{Visual observations}

Discolouration is the first manifestation of degradation, which is due to intermolecular cyclization process. Oxidative degradation causes discolouration and rapid loss of mechanical and dielectric strength (Cherney 1996). Many polymers discolour during aging due to oxidation or functional group elimination along the polymer chain. Severe discolourations were observed in all the irradiated samples

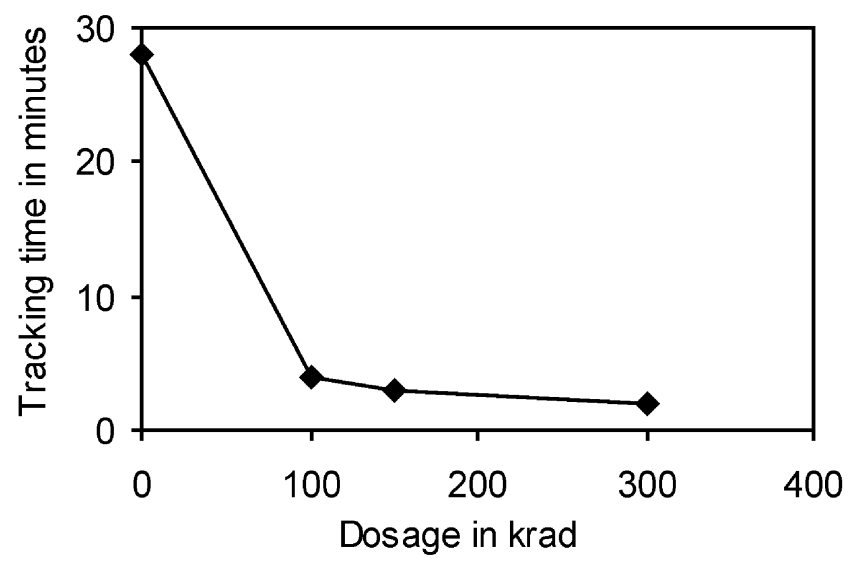

Figure 3. Variation of tracking time with gamma dosage.

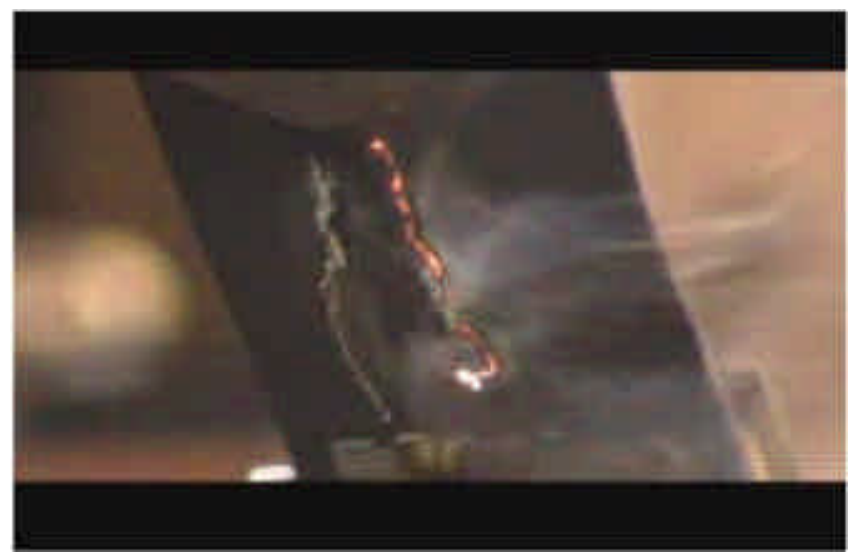

Figure 4. A typical CCD snap shot during tracking. 
after subjecting them to high voltage. These findings are consistent with the findings of previous researchers (Cherney et al 1996; Raji Sundarrajan et al 2004).

\subsection{Estimation of fractal dimension}

The tracking patterns of the irradiated samples were scanned and the images were treated to filter noise. The
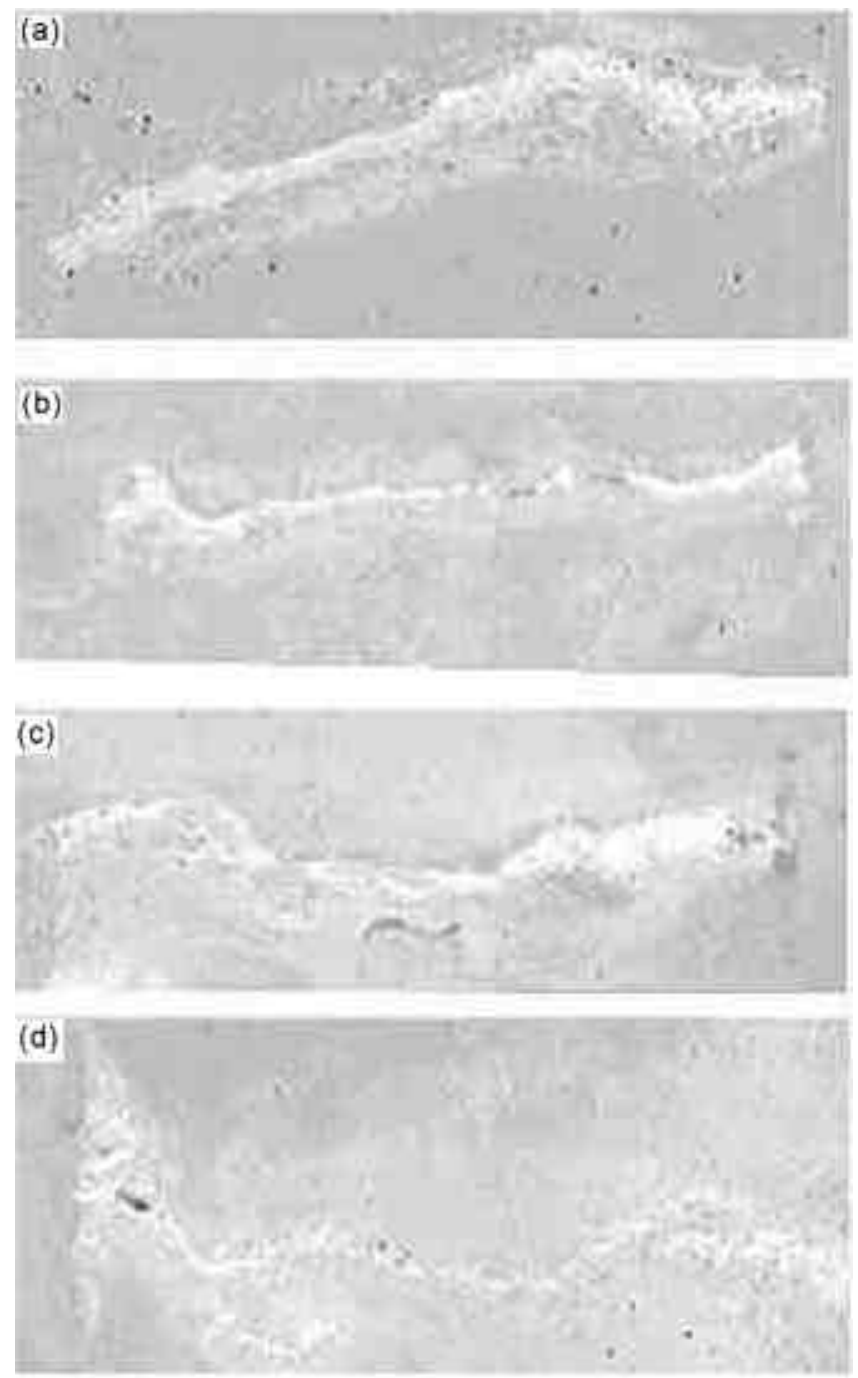

Figure 5. Tracking patterns of (a) virgin, (b) $100 \mathrm{krad,} \mathrm{(c)}$ $150 \mathrm{krad}$ and (d) $300 \mathrm{krad}$ samples.

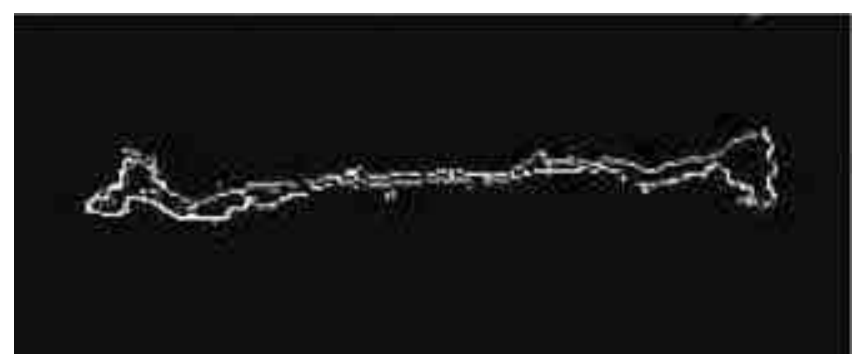

Figure 6. Edge detected pattern of $100 \mathrm{krad}$ sample. tracking patterns are shown in figure 5. The pictures in figure 5 are inverted for better clarity. Using edge detection method, the tracked portion is identified. The edgedetected image of 100-krad pattern is given in figure 6 . After edge detection the tracked portion is filled (see figure 7) and the fractal dimension is calculated using the box counting method. The results are shown in figure 8 .

It could be seen that each tracking pattern is unique and so is the fractal dimension. There is a close correlation between the fractal dimensions of the tracked patterns and their tracking time.

\subsection{SEM analysis}

SEMs are patterned, after reflecting light microscopes and give information on surface topology, morphology and crystallography. The topology gives surface features of an object or how it looks, its texture. The detectable features are limited to a few $\mathrm{nm}$. The morphology gives the shape, size and arrangements of the particles making up the object that are lying on the surface of the sample. Thus, they are useful in identifying the cracks that cannot be seen with unaided eye.

Figure 9 shows SEM micrographs of virgin and irradiated samples. There is a noticeable difference in the surface structure and severity of cracks between virgin and irradiated samples. Figure 9a shows the SEM micrograph of unaged sample at $25 \times$. The surface is smooth and homogeneous. There is no noticeable cracks or other aberrations.

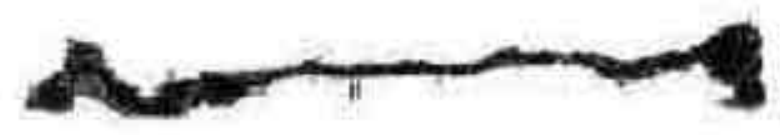

Figure 7. Edge detected pattern of $100 \mathrm{krad}$ sample after filling.

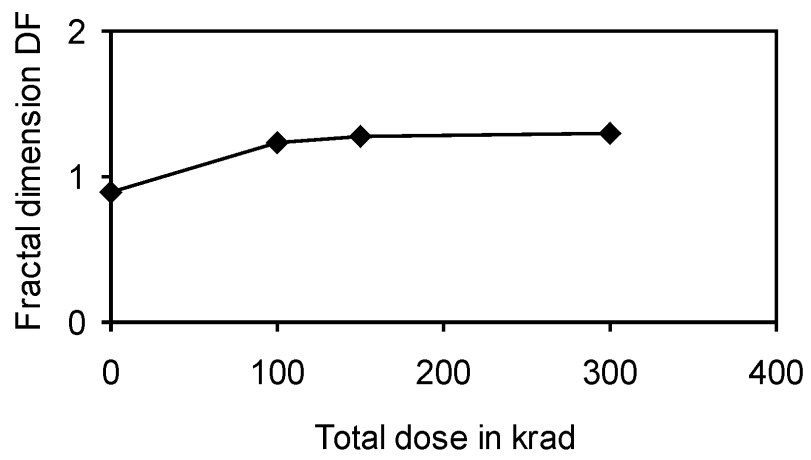

Figure 8. Variation in fractal dimension with gamma dosage. 

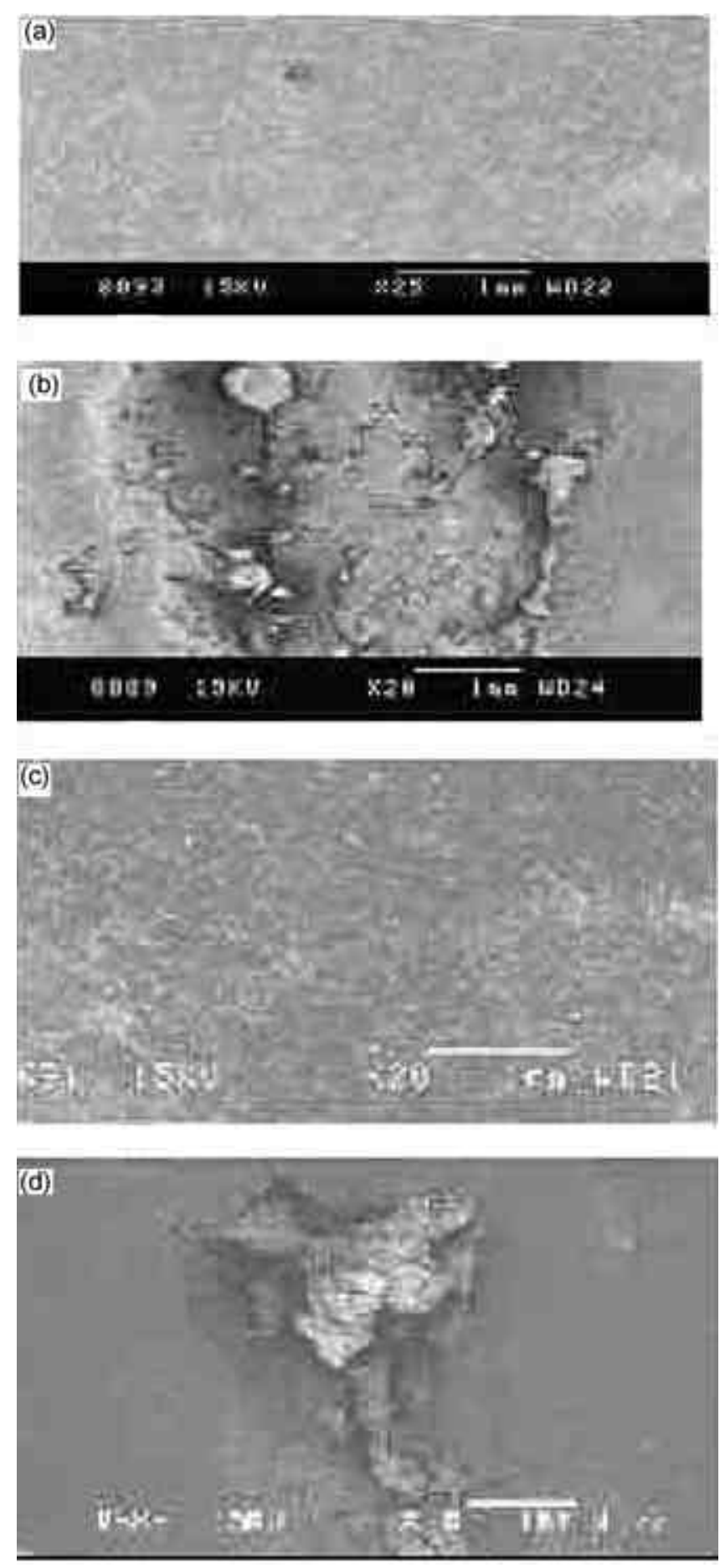

Figure 9. SEM images of (a) virgin sample, (b) virgin and tracked sample, (c) $100 \mathrm{krad}$ irradiated sample and (d) $100 \mathrm{krad}$ irradiated and tracked samples.

Figure $9 \mathrm{~b}$ shows the surface cracking and the loosely bound filler material on the sides. Figure 9c depicts clearly the surface changes that have taken place due to irradiation at $20 \times$. Figure 9d shows the damages due to tracking in irradiated sample. The width of crack is more in virgin samples compared to others.

\section{Conclusions}

In this paper, we have examined the nature of fractal dimensions of tracked patterns. The tracking patterns are found to be unique and fractal dimension ranges from $\sim 0.8972$ for unaged sample to $\sim 1.3083$ for sample aged to $300 \mathrm{krad}$ dosage. The fractal dimension of the track formed in sample aged by $1496 \mathrm{krad}$ was $\sim 1 \cdot 2915$. The experiments in this paper should provide useful guidelines for researchers wishing to use fractal feature for pattern discrimination of tracked images and to quantify the damage caused by tracking. In this short communication, we have investigated and presented preliminary results on the use of fractal dimension for recognition of 2- $d$ tracked patterns. This work is based on the assumption that the 2-d image patterns qualify as a fractal pattern, and found to possess pattern recognition capability. The variation in fractal dimensions with different voltages has to be studied thoroughly. From the limited results on hand, it can be conjectured that this approach appears promising for pattern discrimination and can be used to quantify the damage caused by tracking and further research is necessary before its true potential could be gauged. Moreover, ethylene propylene diene monomer was found to be less suitable for radiation prone environments. This may be due to the overall increase in temperature or localized hotspots within the material, which may eventually reduce the hydrophobicity of the surface and hence accelerate the aging of the sample. The suitability of this material in similar environments with a lower voltage stress and low level ageing needs to be studied.

\section{Acknowledgements}

We express our sincere thanks to Dr Ponraj, and Mr S Siva Kumar, IGCAR, Kalpakkam, who have helped us in irradiating the samples. The first author gratefully acknowledges the financial support provided by S.S.N. College of Engineering for the test set up.

\section{References}

Candela R, Mirolli G and Schifani R 2000 IEEE Trans. Dielec. Electr. Insul. 787

Cherny E A 1996 IEEE Trans. Dielec. Electr. Insul. 245

Fouracre R A, Bandford H M, Tedford, Wang Huming D J and Xialolong Cao 1989 3rd international conference on conduction and breakdown in solid dielectrics $\mathbf{4 6 9}$

Gorur R S, Chang J W and Amburg O G 1990 IEEE Trans. on Power Delivery 41923

IEC Publication 1984 Testing method for evaluating the resistance of tracking and erosion of electrical insulating materials used under severe ambient conditions, No. 587

Kebbabi L and Beroual A 2006 J. Phys. D: Appl. Phys. 39 177

Kudo K 1998 IEEE Trans. Dielec. Electr. Insul. 5713 
Li H M, Fouracre A, Given M J and Banford H M 1999 IEEE Trans. Dielec. Electr. Insul. 6295

Niemeyer L, Pietronero L and Wiesmann H 1984 Phys. Rev. Lett. 521033
Raji Sundarrajan, Areef Mohamed and Nopporn Chaipanit 2004 IEEE Trans. Dielec. Electr. Insul. 11349

Starr W T 1990 IEEE Trans. Dielec. Electr. Insul. 25125

Wang S G 2004 Elsevier Pub., Physica A335 1 